Comparison between Hartree-Fock and Kohn-Sham electronic and structural properties for hexagonal-close-packed magnesium

This content has been downloaded from IOPscience. Please scroll down to see the full text. 1998 J. Phys.: Condens. Matter 1010969

(http://iopscience.iop.org/0953-8984/10/48/017)

View the table of contents for this issue, or go to the journal homepage for more

Download details:

IP Address: 193.54.110.42

This content was downloaded on 23/05/2015 at 13:38

Please note that terms and conditions apply. 


\title{
Comparison between Hartree-Fock and Kohn-Sham electronic and structural properties for hexagonal-close-packed magnesium
}

\author{
I Baraille†, C Pouchan†, M Causàł and F Marinelli§̧ \\ $\dagger$ Laboratoire de Chimie Structurale, UMR 5624, IFR rue Jules Ferry, 64000 Pau, France \\ $\ddagger$ Department CIFM, University of Torino, via Giuria 5, I-10125 Torino, Italy \\ $\S$ Centre de Thermodynamique et Microcalorimetrie 26, rue du $141^{\text {ième }}$ RIA, 13331 Marseille, \\ France
}

Received 16 June 1998, in final form 22 September 1998

\begin{abstract}
The properties of hcp magnesium are investigated using the density functional method with the linear combination of atomic orbitals as implemented in the CRYSTAL95 code. The lattice equilibrium parameters and the binding energy have been calculated at the Hartree-Fock level, at the hybrid Hartree-Fock density functional level, and at the Kohn-Sham density functional level using local and non-local exchange and correlation potentials. The electronic properties (band structures, topologies of the Fermi surface, and densities of states) and the elastic constants are computed for each type of functional, and compared to experimental data.
\end{abstract}

\section{Introduction}

Density functional theory (DFT) is one of the techniques commonly used for studying accurately the electronic structure of atoms and molecules. It has been demonstrated as effective and competitive with other quantum chemical methods [1-3]. For periodic systems, DFT is at present the only suitable technique for treating the electronic correlation with the standard computer programs from general treatment methods because it takes into account explicitly exchange and correlation functionals and potentials. Density functional linear combination of atomic orbitals (LCAO) calculations generally improve the lattice parameters, the thermochemical data, and the elastic behaviour of covalent, ionic, and semi-ionic crystals as compared to pure Hartree-Fock results [4-6]. Little is known concerning metallic systems. Following up our investigations on the hexagonal-close-packed (hcp) magnesium system [7], which has been the subject of extensive theoretical and experimental work [8-13], we have carried out a systematic and comparative study of Hartree-Fock (HF), DFT local density approximation (LDA), and DFT generalized gradient approximation (GGA) calculations on hcp Mg properties. In this work, several forms of the exchange-correlation functional are used to improve the agreement between the calculated and experimental properties and to establish the limits of each approach.

The outline of this paper is as follows. In section 2, methods and computational details are presented. In sections 3, 4, and 5, the results on structural and thermodynamical properties, and of electronic structure and elastic property calculations for $\mathrm{Mg}$ are successively summarized and discussed. 


\section{Computational details}

In the present study, the CRYSTAL95 computer program [14] has been used. It has been generalized in order to apply DFT-LCAO methods as well as the HF-LCAO technique to periodic systems. We refer the reader to previous publications for a description of the periodic Hartree-Fock crystalline orbital LCAO self-consistent-field computational scheme as implemented in this code [4-6]. The DFT method is used at two levels of approximation. In the first level, different correlation functionals have been applied to the Hartree-Fock electronic density in the so-called a posteriori DFT correlation method. This method is referred to as the hybrid HF-DFC/HF method where '/HF' indicates that HF electronic density is used as the input density for the correlation density functional. At the second level of approximation, the exchange and correlation potentials are included in the crystal Hamiltonian. In this case, the Kohn-Sham equations are solved self-consistently and this method is denoted as 'DFX-DFC/DFX-DFC' where the symbols 'DFC' and 'DFX' are substituted for with the appropriate names of the correlation and exchange functionals respectively. The exact exchange-correlation energy functional may be expressed in terms of the electron density, gradients of the density, higher-order derivatives of the electron density, and numerous non-local terms. The local exchange-correlation functional only depends on the electron density, and the non-local corrections correspond to gradients of the density. In our study, we have used a set of local density approximation (LDA) and generalized gradient approximation (GGA) exchange and correlation functionals which gave reasonable results in previous calculations on covalent and ionic crystals $[5,6]$. Local density approximation calculations were performed with the Dirac-Slater exchange (LDA) [15] and the von Barth and Hedin correlation (VBH) [16] energy functionals. The non-local exchange potential given by Becke (BEC) [17] is used while the non-local correlation potential is that given by Perdew (P91) [18].

The Kohn-Sham LCAO periodic method based on numerical integration at each cycle of the self-consistent-field process is computationally more expensive than the periodic LCAO Hartree-Fock method which is almost fully analytical. For this reason, the HF-DFC/HF model can be interesting from a computational point of view if it gives reasonable results. The extension of the existing HF-LCAO CRYSTAL code to a DFT one allows a very direct comparison between these methods using the same code, the same basis set, and the same computational conditions.

Such calculations are affected by two kinds of error: the basis-set incompleteness and the numerical approximations introduced in the implementation of the HF (or KS) equations. The all-electron basis set adopted in this work is similar to that used in previous studies on magnesium compounds [19]: 13 atomic orbitals per atom have been used from linear combinations of Gaussian-type functions (GTF) which are each the product of a radial Gaussian times a real solid harmonic. The basis set can be written as $8-6-11 \mathrm{G}$ where the numbers refer to the level of contraction. The exponents of the last two sp shells have been optimized at the HF level by searching for the minimum crystalline total energy for the experimental geometry. The convergence of the exchange series imposes lower limits on the values of these exponential coefficients. The more the spatial extension of the basis functions increases, the less well the exchange series converges. The two optimal external exponents $(0.100$ and 0.425$)$ take this limit into account. The effects of $\mathrm{d}$ functions optimized to minimize the total energy $\left(\alpha_{\mathrm{d}}=0.234\right)$ have been shown to be negligible at the HFDFC/HF level: such data as lattice parameters and the binding energy are not significantly improved, and elastic constants even become worse. These effects are just analysed in the non-local approximation (BEC-P91). Such a basis set is obviously inadequate for a metal 
system like the present one, but in their HF calculations on hcp beryllium, Dovesi et al [20] showed, trying a number of extended s-p sets and several contraction schemes, that the quality of the basis did not depend as much on the number of independent Gaussian orbitals as on the range of the exponents that they span.

The computational conditions were chosen to minimize the numerical errors introduced in the evaluation of the Coulomb and exchange series as well as in the reciprocal-space integration. The Fock matrix was diagonalized at $133 k$-points within the irreducible Brillouin zone. $793 k$-points are needed to determine the Fermi energy and reconstruct the density matrix by means of a Fourier interpolation from the calculated eigenvalues and eigenvectors. The use of $270 k$-points instead of 793 changes the total energy by about $0.04 \mathrm{eV}$ per atom. The resulting total-energy error propagates through first and second energy derivatives to the lattice parameters, binding energy (BE), and elastic constants. The error bars for these data are about $0.01 \AA, 0.01 \mathrm{eV}$, and $10^{8} \mathrm{~N} \mathrm{~m}^{-2}$ respectively. The equilibrium geometry for each type of calculation is obtained by optimizing simultaneously the two lattice parameters $a$ and $c$ with respect to the crystalline energy by a gradient method (the Newton-Raphson method). All binding energy results include the zero-point vibrational energy (ZPE) and the basis-set superposition error corrections (BSSE). For each optimized geometry, the energy of the free atom has been evaluated by considering one $\mathrm{Mg}$ atom surrounded by 19 ghosts, supporting an adequate basis set and located at the lattice position of the neighbouring atoms.

At each level of approximation, the electronic structure is investigated. The results include Mulliken population analysis, plots of band structures, and density-of-states (DOS) functions. The accuracy of the band structures is evaluated by examining the Fermi surface (FS) in a reduced zone scheme. In an extended zone scheme, for such divalent hcp metals as $\mathrm{Mg}, \mathrm{Zn}$ or $\mathrm{Cd}$, the first double zone has a region not occupied by electrons. This region of holes is called 'the monster'. On the other hand, some electrons occupy several bits of the third and fourth zones which are called the 'pockets of electrons'. Extensive theoretical $[8,21]$ and experimental [22] studies have been made, and no reasonable doubt remains as to the existence and the general topology of these pieces of FS. A comparison of calculated dimensions at different levels of approximation with experimental ones is made.

The technique used to calculate the elastic constants was already described by Catti et al [23]. The total crystalline energy of the system is calculated for a number of different deformations of the unit cell and fitted against the strain component to polynomial functions up to the fourth order. Thus the second derivatives of energy at the minimum can be calculated, and yield the elastic constants.

\section{Structural properties and binding energy}

Table 1 reports the BE for the calculated equilibrium conformation for the different levels of approximation. The relative percentages of the deviation from the experimental data $[24,25]$ for the lattice parameters $(a$ and $c$ ) are reported, to summarize the performance of each method. A correction of $0.04 \mathrm{eV}$ per atom resulting from the zero-point vibrational contribution from the Debye model is included in the binding energy.

The agreement is good for the predicted lattice constants. The relative error on lattice parameter $a$ varies between $-1.9 \%$ and $3.7 \%$ for LDA-VBH and HF calculations, respectively. In all cases, the lattice parameter $c$ is underestimated compared to the extrapolated $0 \mathrm{~K}$ value, by $-3.5 \%$ to $-1 \%$ for the LDA-VBH and HF levels of approximation, respectively. HF calculations are well known to overestimate lattice constants. However, there are some examples showing an underestimation. (The relative error at the HF level 
Table 1. Lattice parameters $(a$ and $c$ ) and binding energies (BE) calculated using exchange and correlation energy-density functionals. The $a=3.19 \AA$ and $c=5.18 \AA$ values correspond to lattice constants extrapolated to $0 \mathrm{~K}$. The relative percentages of the deviations from the experimental data $(a=3.19 \AA$ and $c=5.18 \AA)$ are given in parentheses.

\begin{tabular}{|c|c|c|c|c|c|c|}
\hline & Experiment [24] & $\mathrm{HF}$ & $\begin{array}{l}\text { HF-P91/ } \\
\text { HF }\end{array}$ & $\begin{array}{l}\text { LDA-VBH/ } \\
\text { LDA-VBH }\end{array}$ & $\begin{array}{l}\text { BEC-VBH/ } \\
\text { BEC-VBH }\end{array}$ & $\begin{array}{l}\text { BEC-P91/ } \\
\text { BEC-P91 }\end{array}$ \\
\hline$a(\AA)$ & $\begin{array}{l}3.21 \\
(3.19)\end{array}$ & $\begin{array}{l}3.31 \\
(3.7)\end{array}$ & $\begin{array}{l}3.19 \\
-\end{array}$ & $\begin{array}{l}3.13 \\
(-1.9)\end{array}$ & $\begin{array}{l}3.25 \\
(1.9)\end{array}$ & $\begin{array}{l}3.23 \\
(1.2)\end{array}$ \\
\hline$c(\AA)$ & $\begin{array}{l}5.21 \\
(5.18)\end{array}$ & $\begin{array}{l}5.13 \\
(-1.0)\end{array}$ & $\begin{array}{l}5.03 \\
(-2.9)\end{array}$ & $\begin{array}{l}5.00 \\
(-3.5)\end{array}$ & $\begin{array}{l}5.10 \\
(-1.5)\end{array}$ & $\begin{array}{l}5.12 \\
(-1.2)\end{array}$ \\
\hline$c / a$ & 1.624 & 1.553 & 1.576 & 1.597 & 1.571 & 1.585 \\
\hline $\mathrm{BE}(\mathrm{eV})$ & 1.51 & 0.27 & 1.42 & 1.80 & 1.28 & 1.37 \\
\hline
\end{tabular}

for the $\mathrm{Na}_{2} \mathrm{O}$ lattice parameter is of the order of that for $c(-1 \%)$ ). Both a posteriori and Kohn-Sham DFT models decrease the lattice constants with respect to the HF ones. The LDA-VBH calculations give errors of $-0.06 \AA$ and $-0.18 \AA$ on $a$ and $c$, respectively. Nonlocal models in both cases reduce the error for $a(0.06 \AA$ and $0.04 \AA$ for BEC-VBH and BEC-P91 calculations, respectively) and for $c(-0.08 \AA$ and $-0.06 \AA$ for BEC-VBH and BEC-P91 calculations, respectively). In all cases, the two competing effects on the lattice parameters lead to an underestimated $c / a$ ratio compared to the almost ideal experimental value (1.624). For an ideal hcp structure with the $c / a$ ratio equal to 1.633 , each atom has twelve equivalent nearest neigbours. Any deviation from this value separates these twelve neighbours into two categories and induces an anisotropy which is all the more important as $c / a$ is smaller than 1.633. Although both lattice parameters calculated at the LDA-VBH level are the smallest obtained relatively to the experimental data, the $c / a$ ratio calculated at this level is the best that we obtained. It is worth noting that the inclusion of a $\mathrm{d}$ function in the basis set at the BEC-P91 level improves the $c / a$ ratio from 1.585 to $1.596(a=3.21 \AA$ and $c=5.12 \AA$ ).

As can be expected, the HF binding energy is substantially underestimated compared to the experimental value, confirming the need for the correlation effects. The LDA calculations including the present LDA-VBH ones and the earlier work of Chou and Cohen [9] overestimate the cohesive energy by $19 \%$ and $9 \%$, respectively. The BEC-P91 results are closer to the experimental value. The inclusion of a d function (BEC-P91 $(+d)$ ) leads to $1.43 \mathrm{eV}$. The correlation-only functional HF-P91 performs as well as or even better than Kohn-Sham methods. This compares with the results obtained by Causà and Zupan [5] for some covalent, ionic, and semi-ionic crystals. Indeed, for these compounds the binding energies obtained by the Hartree-Fock LCAO method are more improved by the a posteriori calculations of the electronic correlation than by means of the density functional theory. The minimum deviations calculated by Causà and Zupan are of $-8.2 \%$ and $-18 \%$ for the HF-P91 and BEC-P91 methods respectively. The difference between the HF and KS densities, which is not even detectable for the BE calculations, is investigated by analysing the electronic properties at each level of approximation.

\section{Electronic properties}

All of the electronic properties were evaluated for the optimized geometry at each level of approximation. 
Table 2. Mulliken population data (in units of $|e|$ ) for the valence atomic orbitals (s, $\mathrm{p}_{x}, \mathrm{p}_{y}$, $\left.\mathrm{p}_{z}\right) . z$ is chosen to lie along the $c$-axis of the hcp structure.

\begin{tabular}{llllll}
\hline & HF & LDA-VBH & BEC-VBH & BEC-P91 & BEC-P91 $(+\mathrm{d})^{\mathrm{a}}$ \\
\hline $\mathrm{s}$ & 0.776 & 0.948 & 0.957 & 1.016 & 1.007 \\
$\mathrm{p}_{x}$ & 0.411 & 0.389 & 0.366 & 0.346 & 0.343 \\
$\mathrm{p}_{y}$ & 0.411 & 0.389 & 0.366 & 0.346 & 0.343 \\
$\mathrm{p}_{z}$ & 0.415 & 0.387 & 0.385 & 0.351 & 0.340 \\
\hline
\end{tabular}

${ }^{a}$ The remaining population in the $\mathrm{d}$ shell is equal to $0.023|e|$.

In table 2, we reported the numbers of valence electrons per $\mathrm{Mg}$ atom with $\mathrm{s}, \mathrm{p}_{x}, \mathrm{p}_{y}$ and $\mathrm{p}_{z}$ characters obtained by a Mulliken population analysis ( $z$ is chosen to lie along the $c$-axis of the hcp structure). Table 2 shows the weak anisotropy for the different $\mathrm{p}$ components. The difference between the $\mathrm{p}_{z}$ and $\mathrm{p}_{x}$ (or $\mathrm{p}_{y}$ ) populations is consistent with the departure from the ideal $c / a$ ratio (see table 1). The ratios of $\mathrm{p}_{x}+\mathrm{p}_{y} / \mathrm{p}_{z}$ charges are very close to 2 (completely spherical charge-density distribution), except for BEC-VBH calculations. This was also found by Blaha et al [11] and Chou and Cohen [9] in LAPW and pseudopotential calculations respectively. In the case of the BEC-P91 $(+\mathrm{d})$ method, the remaining population in the $\mathrm{d}$ shell is very low $(0.023|e|)$.

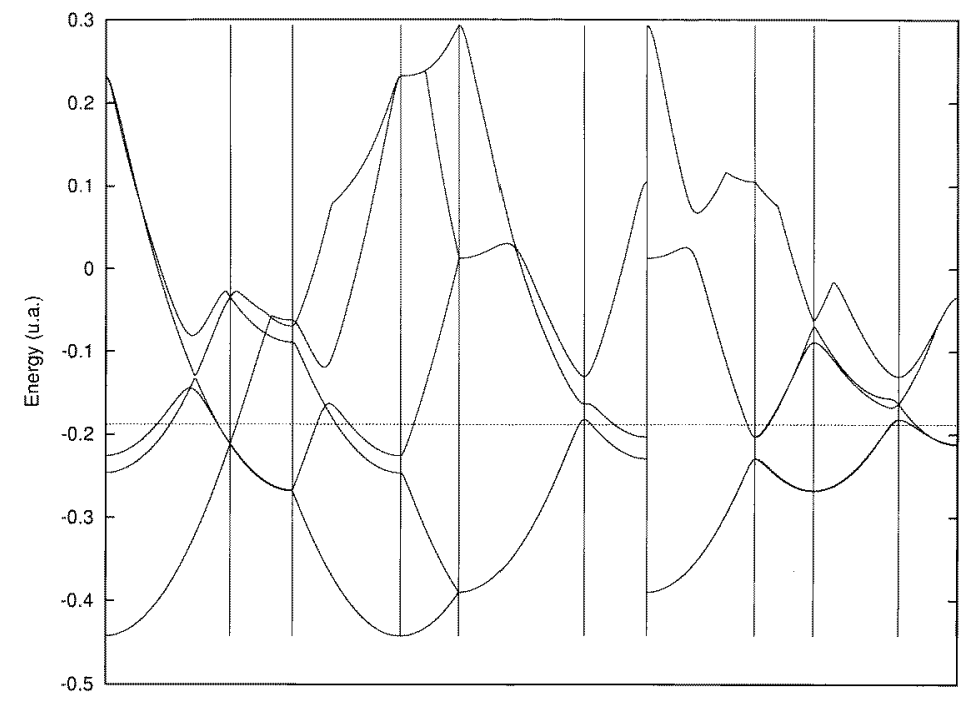

Figure 1. The band structure calculated at the BEC-P91(+d) level of approximation for hcp $\mathrm{Mg}$ along the lines ГКМГAHL and ALMHK in the first Brillouin zone.

At each level of approximation, band structures were obtained along the lines ГКМГАHL and ALMHK in the first Brillouin zone. Except for the case of HF calculations, ours results are very similar to each other and to previous ones [11,21] as far as the topologies of occupied bands are concerned. This means that the FS contains all of the following elements (described by Stark [22]): a first-zone pocket around $\mathrm{H}$ (cap); a secondzone hole (monster); third-zone electrons around $\Gamma$ (lens), around L (butterfly), and around $\mathrm{K}$ (needles); and a fourth-zone electron pocket around L. As an example of a topology, 
Table 3. Calculated Fermi surface dimensions $\left(\mathrm{in}^{\mathrm{au}}{ }^{-1}\right.$ ) and conduction bandwidths (from the bottom up the Fermi level) $\Delta_{F}$ (in eV) compared to experimental, free-electron data, and LMTO results [21]. (1) First-band holes. (2) Second-band monsters. (3a) Third-band needles. (3b) Third-band butterflies. (3c) Third-band lenses. (4) Fourth-band cigars.

\begin{tabular}{|c|c|c|c|c|c|c|c|c|c|}
\hline & & $\begin{array}{l}\text { Experiment } \\
{[22]}\end{array}$ & Free & $\begin{array}{l}\text { LMTO } \\
{[21]}\end{array}$ & $\mathrm{HF}$ & LDA-VBH & BEC-VBH & BEC-P91 & BEC-P91 $(+d)$ \\
\hline \multirow[t]{3}{*}{ (1) } & HA & - & 0.042 & 0.029 & Absent & 0.024 & 0.021 & 0.019 & 0.029 \\
\hline & HL & - & 0.096 & 0.039 & Absent & 0.024 & 0.018 & 0.029 & 0.039 \\
\hline & HK & - & 0.107 & 0.071 & Absent & 0.053 & 0.035 & 0.056 & 0.074 \\
\hline \multirow[t]{7}{*}{ (2) } & $\Sigma \mathrm{L}$ & 0.064 & 0.112 & 0.063 & - & - & - & - & - \\
\hline & HA & - & 0.142 & 0.029 & Absent & 0.024 & 0.018 & 0.029 & 0.039 \\
\hline & HL & - & 0.096 & 0.039 & Absent & 0.024 & 0.018 & 0.029 & 0.039 \\
\hline & $\Gamma \mathrm{M}_{\mathrm{in}}$ & 0.370 & 0.341 & 0.369 & 0.378 & 0.375 & 0.353 & 0.354 & 0.357 \\
\hline & $\Gamma K_{\text {in }}$ & 0.370 & 0.341 & 0.369 & 0.380 & 0.372 & 0.351 & 0.355 & 0.357 \\
\hline & $\Gamma \mathrm{M}_{\text {out }}$ & 0.476 & 0.477 & 0.469 & 0.392 & 0.473 & 0.453 & 0.459 & 0.466 \\
\hline & $\Gamma \mathrm{K}_{\text {out }}$ & 0.622 & 0.634 & 0.621 & 0.606 & 0.632 & 0.597 & 0.611 & 0.622 \\
\hline \multirow[t]{2}{*}{ (3a) } & $\mathrm{K} \Gamma$ & 0.067 & 0.061 & 0.062 & 0.165 & 0.076 & 0.076 & 0.071 & 0.067 \\
\hline & KM & 0.033 & 0.032 & 0.036 & 0.048 & 0.040 & 0.043 & 0.039 & 0.036 \\
\hline \multirow[t]{4}{*}{$(3 b)$} & $\mathrm{L} \Sigma$ & 0.206 & 0.252 & 0.200 & - & - & - & - & - \\
\hline & LH & 0.184 & 0.252 & 0.200 & - & 0.156 & 0.177 & 0.176 & 0.179 \\
\hline & LM & - & 0.087 & 0.082 & Absent & 0.056 & 0.058 & 0.069 & 0.073 \\
\hline & LA & - & 0.051 & 0.047 & Absent & 0.034 & 0.038 & 0.040 & 0.041 \\
\hline \multirow[t]{3}{*}{ (3c) } & $\Gamma A$ & 0.080 & 0.085 & 0.084 & 0.023 & 0.072 & 0.063 & 0.068 & 0.072 \\
\hline & $\Gamma \mathrm{M}$ & 0.312 & 0.341 & 0.320 & 0.119 & 0.290 & 0.265 & 0.274 & 0.283 \\
\hline & $\Gamma \mathrm{K}$ & 0.312 & 0.341 & 0.320 & 0.119 & 0.291 & 0.265 & 0.275 & 0.284 \\
\hline \multirow[t]{4}{*}{ (4) } & LA & - & 0.051 & 0.047 & Absent & 0.034 & 0.038 & 0.040 & 0.040 \\
\hline & LM & 0.043 & 0.087 & 0.080 & Absent & 0.056 & 0.058 & 0.069 & 0.073 \\
\hline & LH & - & 0.252 & 0.198 & Absent & 0.156 & 0.177 & 0.176 & 0.179 \\
\hline & $\Delta_{F}$ & $6.1[26]$ & 7.13 & - & & 7.40 & 7.01 & 6.94 & 6.91 \\
\hline
\end{tabular}

figure 1 shows the band structure obtained at the BEC-P91 $(+\mathrm{d})$ level of approximation. The band structures only differ in the quantitative dimensions of various elements of the FS and in the value of the conduction bandwidth (from the bottom up to the Fermi level) (see table 3). It should be noted that the HF method clearly failed to give a correct picture of the electronic band structure of Mg. Dovesi et al [20] have shown, in the hep Be case, that the HF band structure is very much dependent on the computational conditions (lattice parameters and basis set). For the DFT methods, our results are in quantitative agreement with the experimental ones. It can be observed however that these results are dependent on the lattice parameters. For the same geometry, calculations at the BEC-P91 level with or without $\mathrm{d}$ functions lead to approximately the same results to within about $2 \%$. The slightly different values that we reported for $\Gamma \mathrm{M}_{\text {in }}$ and $\Gamma \mathrm{K}_{\text {in }}$ lines in the secondband monster are due to the small anisotropy induced by the values of some optimized $c / a$ ratios. Except for the LA line in the fourth-zone cigar and the KM one in the thirdband needles, the worst deviations from experiment are 15\% for LDA-VBH and BEC-P91, $13.5 \%$ for BEC-P91 $(+\mathrm{d})$, and $9 \%$ for LMTO calculations [21]. It is worth noting that there is no difference between the results for the fourth-zone electron pockets and the butterflies 
along the LM and LA lines. This may be attributable to the fact that our calculations did not include the spin-orbit coupling effect. As in previous calculations, our calculated bandwidths $\Delta_{F}$ are overestimated compared to the experimental value $(6.1 \mathrm{eV})$ measured by photoelectron spectroscopy [26]. Hufner [27] has suggested that this discrepancy is attributable to the photoelectron process; the data must be corrected in the framework of Mahan-Nozières-DeDominicis theory. However, the corrected value that Hufner proposed $(6.2 \mathrm{eV})$ remains smaller than those obtained in our calculations and in the free-electron approximation $(7.13 \mathrm{eV})$.

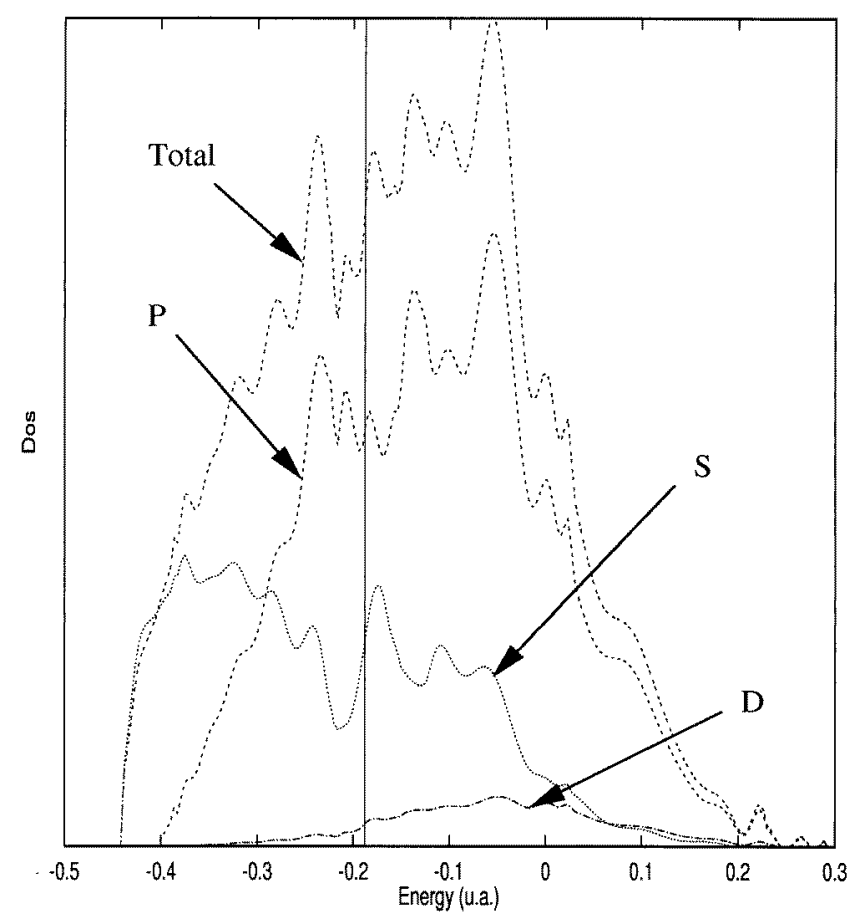

Figure 2. Total and projected densities of states at the BEC-P91 $(+d)$ level of approximation. The Fermi level is shown by a vertical line.

Total and projected densities of states (DOS) at the BEC-P91 $(+\mathrm{d})$ level are given in figure 2. The very low contribution of $d$ orbitals in the occupied part of the conduction band is clearly shown, and confirms the negligible role played by $\mathrm{d}$ functions in our computed electronic densities. The DOSs obtained with the other DFT methods are very similar, and compare very well with the previous ones [9, 21]. At the bottom of the conduction band, the total DOS functions exhibit a parabolic dependence characteristic of the free-electron gas, and have essentially a s-like contribution. At the top, below the Fermi level $\left(\epsilon_{F}\right)$, they are disturbed by the lattice potential, and have an increasing $\mathrm{p}$ contribution. Our results relating to $\mathrm{s}$ and $\mathrm{p}$ contributions are in agreement with the APW densities of states obtained by Gupta and Freeman [28], but their predicted d contribution is found to be very important (about 30\% at $\epsilon_{F}$ ). On the other hand, the results of Citrin et al [13] did not include any d contribution, but they are dominated by the $s$ density of states even just below $\epsilon_{F}$. These results were found to be in better agreement with experimental data, especially in the region close to the Fermi level, than those given by Gupta and Freeman [29]. 


\section{Elastic constants}

The six independent components of the elastic tensor were obtained as follows. $C_{11}$ and $C_{33}$ were computed directly by deforming the $a$ - and $c$-edges of the primitive cell. $C_{12}$ and $C_{13}$ were calculated indirectly via the linear combinations $C_{11}+C_{12}$ and $C_{11}+C_{12}+2 C_{33}+0.5 C_{13}$ from a strain involving the deformation of the two $a$-sides and of the three $a$-sides, respectively. The shear constant $C_{44}$ is obtained from the deformation of the $\alpha$-angle. $C_{66}$ and the bulk modulus are computed from the formulae $0.5\left(C_{11}-C_{12}\right)$ and $(1 / 9)\left(2 C_{11}+2 C_{12}+4 C_{33}+C_{13}\right)$.

Table 4. Elastic constants (in units of $10^{11} \mathrm{~N} \mathrm{~m}^{-2}$ ) and bulk moduli $B$ (in GPa) calculated at each level of approximation; comparison with experimental data [30].

\begin{tabular}{lllllll}
\hline & Experiment & HF & HF-P91 & BEC-VBH & LDA-VBH & BEC-P91(+d) \\
\hline$C_{11}$ & 0.6347 & 0.933 & 0.924 & 0.647 & 0.787 & 0.637 \\
$C_{33}$ & 0.6645 & 1.023 & 1.435 & 0.708 & 0.947 & 0.682 \\
$C_{44}$ & 0.1863 & - & - & 0.162 & 0.110 & 0.190 \\
$C_{66}$ & 0.1878 & 0.429 & 0.391 & 0.247 & 0.259 & 0.242 \\
$C_{12}$ & 0.2594 & 0.028 & 0.143 & 0.153 & 0.269 & 0.152 \\
$C_{13}$ & 0.2170 & 0.096 & 0.067 & 0.208 & 0.246 & 0.241 \\
$B$ & 36.90 & 36.99 & 42.64 & 34.88 & 44.92 & 35.89 \\
\hline
\end{tabular}

The calculated elastic constants are reported in table 4, together with experimental data [30]. The HF model failed to give reasonable elastic constants. $C_{11}$ and $C_{33}$ are too high by approximately $50 \%$ compared with the experimental values while $C_{12}$ and $C_{13}$ are too small. The good agreement between our bulk modulus and the experimental value is clearly fortuitous. The a priori DFT approximation does not improve these results except for the $C_{12}$-value. Kohn-Sham models more correctly reproduce the elastic constants, even if the relative error on the LDA-VBH values is found to be about $40 \%$ for $C_{33}, C_{44}$, and $C_{66}$, and $20 \%$ for $C_{11}$. The agreement between our BEC-VBH and BEC-P91 $(+\mathrm{d})$ calculated values and the experimental data is quite satisfactory (within $2 \%$ for $C_{11}, 7 \%$ for $C_{33}$, and $13 \%$ for $C_{44}$ ). However, the calculated $C_{12}$-values are about $40 \%$ smaller than the experimental one, and this explains the error of $30 \%$ on the $C_{66}$-values. As a general rule, the off-diagonal elastic constants such as $C_{12}$ are affected by larger errors, and the agreement noted for the $C_{13}$-values (within $12 \%$ ) can be considered surprising. On the other hand, the LDA-VBH approximation gives underestimated lattice parameters, producing a too-small equilibrium volume of the cell $\left(42.45 \AA^{3}\right.$ instead of $\left.45.65 \AA^{3}\right)$, while the BEC-VBH and BEC-P91 $(+\mathrm{d})$ ones lead to values close to the experimental ones (46.65 and $45.53 \AA^{3}$, respectively).

The bulk modulus values obtained for BEC-VBH (34.88 GPa) and BEC-P91 $(+\mathrm{d})$ $(35.89 \mathrm{GPa})$ calculations are better than our previous values deduced from a first-principles pseudopotential recently published [7] (39.0 and $39.3 \mathrm{GPa}$ )

\section{Conclusions}

We have calculated various properties of magnesium in the hcp structure using the LCAO approach which is generally believed to be inadequate for nearly free-electron systems, within Hartree-Fock or density functional schemes. The results reported in this paper show that a number of properties, especially electronic ones, can be accounted for within DFT-LCAO methods. The standard basis set used for ionic compounds provides a correct 
description of the metallic character of the system, especially when non-local correlation and exchange potentials are used. However, the inclusion of $\mathrm{d}$ functions noticeably improves the calculated results.

\section{References}

[1] Becke A D 1992 J. Chem. Phys. 962155

[2] Gill P M W, Johnson B G, Pople J A and Frisch M J 1992 Chem. Phys. Lett. 197499

[3] Murray C W, Laming G J, Handy N C and Amos R D 1992 Chem. Phys. Lett. 199551

[4] Pisani C 1996 Quantum Mechanical ab initio Calculations of the Properties of Crystalline Materials (Heidelberg: Springer)

[5] Causà M and Zupan A 1994 Chem. Phys. Lett. 220145

Causà M and Zupan A 1994 Int. J. Quantum Chem. Symp. 28633

[6] Zupan A and Causà M 1995 Int. J. Quantum Chem. 56337

[7] Marinelli F, Roche M, Baraille I and Pouchan C 1996 Phys. Rev. B 546054

[8] Falicov L M 1962 Phil. Trans. A 25555

[9] Chou M Y and Cohen M L 1986 Solid State Commun. 57785

[10] Radwan A M and Saleh A A 1987 Fizika 117

[11] Blaha P, Schwarz K and Dederichs P H 1988 Phys. Rev. B 389368

[12] Althoff J D, Allen P B, Wentzcovitch R M and Moriarty J A 1993 Phys. Rev. B 4813253

[13] Citrin P H, Wertheim G K and Baer Y 1977 Phys. Rev. B 164256

Citrin P H, Wertheim G K and Schluter M 1979 Phys. Rev. B 203067

[14] Dovesi R, Saunders V R, Roetti C, Causà M, Harrison N M, Orlando R and Aprà E 1996 CRYSTAL95: User's Manual (University of Torino)

[15] Dirac P A M 1930 Proc. Camb. Phil. Soc. 26376

[16] von Barth U and Hedin L 1972 J. Phys. C: Solid State Phys. 51629

[17] Becke A D 1988 Phys. Rev. A 383098

[18] Perdew J P 1991 Electronic Structure of Solids 1991 ed P Ziesche and H Eschrig (Berlin: Akademie) pp 11-20

[19] Causà M, Dovesi R, Pisani C and Roetti C 1986 Phys. Rev. B 331308

[20] Dovesi R, Pisani C, Ricca F and Roetti C 1982 Phys. Rev. B 253731

[21] Daniuk S, Jarlborg T, Kontrym-Sznajd G, Majsnerowski J and Stachowiak H 1989 J. Phys.: Condens. Matter 18397

[22] Ketterson J B and Stark R W 1967 Phys. Rev. 156748 Stark R W 1967 Phys. Rev. 162589

Kimball J C, Stark R W and Mueller F M 1967 Phys. Rev. 162600

[23] Catti M, Dovesi R, Pavese A and Saunders V R 1991 J. Phys.: Condens. Matter 34151

Catti M, Pavese A, Dovesi R, Roetti C and Causà M 1991 Phys. Rev. B 443509

[24] Koster W and Franz H 1961 Metall. Rev. 61

[25] Kittel C 1976 Introduction to Solid State Physics 5th edn (New York: Wiley)

[26] Plummer E W 1985 Surf. Sci. 152+153 162

[27] Hufner S 1986 Solid State Commun. 59639

[28] Gupta R P and Freeman A J 1976 Phys. Rev. Lett. 361194

[29] Davies M, Hannah P H, Weightman P and Andrews P T 1984 J. Phys. F: Met. Phys. 14355

[30] Squires G L 1966 Proc. R. Soc. 88919 